\title{
The KNOW-CKD Study: What we have learned about chronic kidney diseases
}

\author{
Kook-Hwan $\mathrm{Oh}^{1 \text { (i) }}$, Minjung Kang ${ }^{1(\mathbb{D})}$, Eunjeong Kang ${ }^{2(\mathbb{D})}$, Hyunjin Ryu ${ }^{1(\mathbb{D})}$, Seung Hyeok Han ${ }^{3(\mathbb{D}}$, Tae-Hyun Yoo $^{3(\mathbb{D})}$,

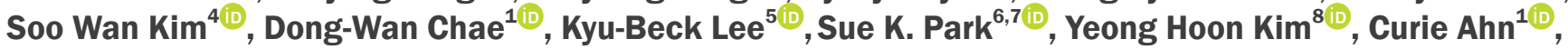 \\ for the KNOW-CKD Study Investigators \\ 'Department of Internal Medicine, Seoul National University College of Medicine, Seoul, Republic of Korea \\ ${ }^{2}$ Department of Internal Medicine, Ewha Womans University Seoul Hospital, Ewha Womans University College of Medicine, Seoul, \\ Republic of Korea \\ ${ }^{3}$ Department of Internal Medicine, College of Medicine, Institute of Kidney Disease Research, Yonsei University, Seoul, Republic of \\ Korea \\ ${ }^{4}$ Department of Internal Medicine, Chonnam National University Medical School, Gwangju, Republic of Korea \\ ${ }^{5}$ Division of Nephrology, Department of Internal Medicine, Kangbuk Samsung Hospital, Sungkyunkwan University School of Medicine, \\ Seoul, Republic of Korea \\ ${ }^{6}$ Department of Preventive Medicine, Seoul National University College of Medicine, Seoul, Republic of Korea \\ ${ }^{7}$ Seoul National University Cancer Research Institute, Seoul, Republic of Korea \\ ${ }^{8}$ Department of Internal Medicine, Inje University Busan Paik Hospital, Inje University College of Medicine, Busan, Republic of Korea
}

\begin{abstract}
As the nation's largest chronic kidney disease (CKD) cohort, the KoreaN Cohort Study for Outcomes in Patients With Chronic Kidney Disease (KNOW-CKD) was established to investigate the clinical course, risk factors for progression, and adverse outcomes of CKD. From 2011 to 2016, the KNOW-CKD recruited 2,238 adult patients with CKD from stage G1 to G5 who were not receiving renal replacement therapy from nine tertiary care hospitals throughout Korea. As of 2019, the KNOW-CKD has published more than 50 articles in the areas of socio-economics, nutrition, quality of life, health-related habits, CKD progression, cardiovascular comorbidity and outcome, anemia, mineral bone disease, biomarker discovery, and international and inter-ethnic comparisons. The KNOW-CKD will eventually offer a prediction model for long-term consequences of CKD, such as the occurrences of end-stage renal disease, cardiovascular disease, and death, thereby enabling the identification and treatment of at-risk populations that require extra medical attention.
\end{abstract}

Keywords: Chronic kidney disease, Cohort studies, Korea, Outcome

Received March 23, 2020; Revised April 22, 2020;

Accepted April 24, 2020

Edited by Eun Hui Bae, Chonnam National University, Gwangju,

Republic of Korea

Correspondence: Kook-Hwan Oh

Department of Internal Medicine, Seoul National University Hospital, 101 Daehak-ro, Jongno-gu, Seoul 03080, Republic of Korea. E-mail: ohchris@hanmail.net

Copyright (C) 2020 by The Korean Society of Nephrology

(a) This is an open-access article distributed under the terms of the Creative Commons Attribution Non-Commercial License (http://creativecommons. org/licenses/by-nc-nd/4.0/), which permits unrestricted non-commercial use, distribution, and reproduction in any medium, provided the original work is properly cited.

\section{Introduction}

Chronic kidney disease (CKD) is a growing health burden worldwide, as well as in Korea [1]. The numbers of patients with CKD and end-stage renal disease (ESRD) are increasing rapidly $[2,3]$. Not only CKD is a significant factor in morbidity and mortality, but the medical expenses for management of CKD are increasing remarkably.

In order to broaden knowledge regarding the risk factors for CKD progression and adverse outcomes, the KoreaN Cohort Study for Outcomes in Patients With 
Chronic Kidney Disease (KNOW-CKD) was established with the support of the Korea Center for Disease Control and Prevention (KCDC) [4]. The study protocol was approved by the ethical committee at each participating center (Supplementary materials, available online). From 2011 until 2016, the KNOW-CKD recruited 2,238 adult patients with CKD from stage G1 to G5 who were not receiving renal replacement therapy from nine tertiary care hospitals throughout Korea [5]. All the subjects being ethnic Koreans, the KNOW-CKD is expected to provide high-level evidence from Koreans on the clinical courses and clinical and biological factors for CKD progression and/or adverse outcomes, and diverse deficits related to CKD such as cardiovascular comorbidity, anemia, mineral metabolic derangements, quality of life (QOL), and health habits. Unlike other CKD cohort studies such as the Chronic Renal Insufficiency Cohort (CRIC) of the United States $[6,7]$ or the Chronic Kidney Disease Japan Cohort (CKD-JAC) of Japan [8], the KNOW-CKD study includes CKD at early stages (stages G1 and G2) as well as advanced stages, providing a platform on which to investigate the courses of early CKD over the long term.

As of December 2019, the KNOW-CKD has published over 50 articles in international peer-reviewed journals. The present article provides the basic design and summary of the major findings from the KNOW-CKD study.

\section{Study design, recruitment, and follow-up status}

Details of the rationale and design of the KNOW-CKD study have been described previously [4]. Briefly, for Phase I of the KNOW-CKD study, 2,238 subjects with CKD were recruited from nine university-affiliated tertiary-care hospitals throughout Korea from 2011 until 2016. Nephrologists, epidemiologists, specialists in laboratory medicine, and biostatisticians have participated in this study. The study is funded and supervised by the KCDC. The protocol summary is also registered at the ClinicalTrials.gov with accession number NCT01630486. The participants were all ethnic Koreans, aged between 20 and 75 years, with CKD stages from G1 to G5 (pre-dialysis). CKD stages were defined on the basis of the estimated glomerular filtration rate (eGFR) calculated using the CKD Epidemiology Collaboration (CKD-EPI) (cr) formula [9]. The serum creatinine was measured at a central laboratory (LabGenomics, Seongnam, Korea), using an assay traceable to the international reference material.

All enrolled subjects were categorized into the following subgroups: glomerular diseases (GN), diabetic nephropathy (DN), hypertensive nephropathy (HTN), polycystic kidney disease (PKD), and unclassified, based on the etiology of CKD. HTN and PKD were oversampled in order to compare the four major causative diseases of CKD. The clinical information and laboratory tests performed at each study visit are depicted in Table 1 [5].

Composite renal events were defined as doubling of serum creatinine, eGFR halving, or development of ESRD. ESRD was defined as starting maintenance dialysis for more than three months or receiving kidney transplantation. CV events were defined as any first event of the following: acute myocardial infarction, unstable angina, ischemic or hemorrhagic cerebral stroke, congestive heart failure, symptomatic arrhythmia, aggravated valvular heart disease, pericardial disease, abdominal aortic aneurysm, and severe peripheral arterial disease that required hospitalization, interventions, or therapy during follow-up. Data on the time and specific causes of mortality were obtained either from hospital records or from the Korean Statistical Information Service (KOSIS). Other events such as all-cause hospitalization, surgery, and fracture were also collected during the follow-up period. All the events described above were recorded on a special report form and further adjudicated by the Central Adjudication Committee of the KNOW-CKD study.

The baseline characteristics of the study subjects are described in Table 2.

As of March 31, 2019, among 2,238 subjects enrolled in the study, there were 107 deaths and 165 fatal or nonfatal CV events after 5.0-year (median) follow-up. Five hundred thirty three subjects developed ESRD (Table 3, Fig. 1).

We subsequently summarize the major findings of the KNOW-CKD study in several areas: 1) socio-economics and health-related habits, 2) CV comorbidity, 3) anemia, 4) mineral bone disease, 5) nutrition and QOL, 6) biomarker discovery studies, 7) clinical courses and outcomes, and 8) international comparison.

\section{Socio-economics and health-related habits}

Socio-economic status (SES), defined by educational attainment and income levels, contributes to a complex set 
Table 1. Sequence and schedule of KNOW-CKD study clinic visits and procedures

\begin{tabular}{|c|c|c|c|c|c|c|c|c|c|c|c|c|c|}
\hline Parameter & Screen & At entry & $6 \mathrm{mo}$ & $1 \mathrm{yr}$ & $2 \mathrm{yr}$ & $3 \mathrm{yr}$ & $4 \mathrm{yr}$ & $5 \mathrm{yr}$ & $6 \mathrm{yr}$ & $7 \mathrm{yr}$ & $8 \mathrm{yr}$ & $9 \mathrm{yr}$ & $10 \mathrm{yr}$ \\
\hline Informed consent & $\bullet$ & & & & & & & & & & & & \\
\hline $\begin{array}{l}\text { Demographic information, Medical } \\
\text { History }\end{array}$ & $\bullet$ & & & & & & & & & & & & \\
\hline Recent events & & & $\bullet$ & $\bullet$ & $\bullet$ & $\bullet$ & $\bullet$ & $\bullet$ & $\bullet$ & $\bullet$ & $\bullet$ & $\bullet$ & $\bullet$ \\
\hline Medications & & $\bullet$ & $\bullet$ & $\bullet$ & $\bullet$ & $\bullet$ & $\bullet$ & $\bullet$ & $\bullet$ & $\bullet$ & $\bullet$ & $\bullet$ & $\bullet$ \\
\hline HRQOL/Health related questionnaire & & $\bullet$ & & & & & & $\bullet$ & & & & & \\
\hline $\begin{array}{l}\text { CBC, chemistry, } \mathrm{Cr}^{\mathrm{a}} \text {, eGFR, Cystatin } \mathrm{C}^{\mathrm{a}} \text {, } \\
\text { HbA1c }\end{array}$ & & $\bullet$ & $\bullet$ & $\bullet$ & $\bullet$ & $\bullet$ & $\bullet$ & $\bullet$ & $\bullet$ & $\bullet$ & $\bullet$ & $\bullet$ & $\bullet$ \\
\hline Lipid panel, CRP, iron panel & & $\bullet$ & & & & $\bullet$ & & & & $\bullet$ & & & \\
\hline Intact PTH ${ }^{\mathrm{a}}, 25 \mathrm{D}^{\mathrm{a}}, 1,25 \mathrm{D}^{\mathrm{a}}$, troponin $\mathrm{T}^{\mathrm{a}}$ & & $\bullet$ & & & & $\bullet$ & & & & $\bullet$ & & & \\
\hline UA with microscopy & & $\bullet$ & & & & $\bullet$ & & & & $\bullet$ & & & \\
\hline ECG, chest $X$ ray & & $\bullet$ & & & $\bullet$ & & & & $\bullet$ & & & & $\bullet$ \\
\hline $\begin{array}{l}\text { EchoCG, PWV, ABI, Coronary CT, LS } \\
\text { spine lateral X-ray, DEXA BMD }\end{array}$ & & $\bullet$ & & & & & $\bullet$ & & & & & & \\
\hline Biosamples for DNA & & $\bullet$ & & & & & & & & & & & \\
\hline Serum/urine biosample & & $\bullet$ & $\bullet$ & $\bullet$ & $\bullet$ & & $\bullet$ & & $\bullet$ & & $\bullet$ & & $\bullet$ \\
\hline Fundus exam (DM subgroup) & & $\bullet$ & & & & & & & & & & & \\
\hline Abdomen CT (PKD subgroup) & & $\bullet$ & & & $\bullet$ & & $\bullet$ & & $\bullet$ & & $\bullet$ & & $\bullet$ \\
\hline Family screen (PKD subgroup) & & $\bullet$ & & & & & & & & & & & \\
\hline
\end{tabular}

1,25 D, 1,25-dihydroxyvitamin D; 24HU, 24-hour urine; 25 D, 25-hydroxyvitamine D; ABI, ankle-brachial index; BP, blood pressure; CBC, complete blood count; Cr, creatinine; CRP, C-reactive protein; CT, computed tomography; DEXA BMD, dual energy x-ray absorptiometry bone mineral density; DM, diabetes mellitus; ECG, electrocardiogram; eGFR, estimated glomerular filtration rate; HbA1c, hemoglobin A1c; HRQOL, health-related quality of life; KNOW-CKD, KoreaN Cohort Study for Outcomes in Patients With Chronic Kidney Disease; LS, lumbosacral; PKD, polycystic kidney disease; PTH, parathyroid hormone; PWV, pulse wave velocity; UA, urinalysis.

${ }^{a}$ Measured at the central laboratory.

of socio-economic determinants, including medical insurance, transportation, stress level, housing quality, and accessibility to medical care [10]. Such determinants may interact and combine to affect health outcomes. Among the KNOW-CKD participants, lower SES was significantly associated with left ventricular hypertrophy, which is a major predictor of future CV outcomes [11]. Currently, analysis of longitudinal follow-up data is underway and is expected to reveal whether lower SES is associated with the risk for CKD progression or poorer $\mathrm{CV}$ outcomes.

While increased body mass index (BMI) is a wellestablished CVD risk factor in the general population, it is paradoxically associated with survival benefits in CKD and ESRD patients $[12,13]$. In contrast to BMI, anthropometric indices representing central obesity such as waist-to-hip ratio (WHR) and waist circumference (WC) are suggested to be directly associated with CVD and mortality. The KNOW-CKD subjects were categorized into 6 groups according to a combination of BMI (normal, overweight, obese) and WHR (lower or higher than sexspecific median WHR). Here, the normal BMI and higher WHR group had the highest risk of coronary artery calcification (CAC) compared with the normal BMI and lower WHR group (odds ratio, 2.104; 95\% confidence interval [CI], 1.074-4.121). Thus, BMI and WHR-two predictors of obesity-can help to discriminate CVD risk in Korean CKD patients [14].

The KNOW-CKD investigated the effects of health habits on CKD outcome. The study collected detailed information on exercise, alcohol drinking, and smoking through self-reported questionnaires. Compared with non-drinkers, regular and occasional binge drinkers had 
Table 2. Baseline demographic and clinical characteristics of KNOW-CKD subjects at study entry

\begin{tabular}{|c|c|c|c|c|c|c|}
\hline Cause of CKD & Total $(n=2,238)$ & $\mathrm{GN}(\mathrm{n}=810)$ & $\mathrm{DN}(\mathrm{n}=519)$ & $\operatorname{HTN}(n=409)$ & $\operatorname{PKD}(n=364)$ & $P$ \\
\hline Age (yr) & $53.7 \pm 12.2$ & $49.8 \pm 12.1$ & $59.3 \pm 9.4$ & $59.6 \pm 10.8$ & $47 \pm 10.6$ & $<0.001^{*}$ \\
\hline \multicolumn{7}{|l|}{ Sex } \\
\hline Male & $1,369(61.2)$ & $451(55.7)$ & $357(68.8)$ & $295(72.1)$ & $184(50.5)$ & \multirow[t]{2}{*}{$<0.001^{\dagger}$} \\
\hline Female & $869(38.8)$ & $359(44.3)$ & $162(31.2)$ & $114(27.9)$ & $180(49.5)$ & \\
\hline \multicolumn{7}{|l|}{ Educational attainment } \\
\hline Illiteracy & $15(0.8)$ & $6(0.9)$ & $1(0.2)$ & $5(1.4)$ & $2(0.6)$ & \multirow[t]{5}{*}{$<0.001^{\dagger}$} \\
\hline Graduated elementary school & $239(10.8)$ & $56(8.0)$ & $71(16.0)$ & $53(14.7)$ & $19(5.6)$ & \\
\hline Graduated middle school & $259(11.7)$ & $62(8.9)$ & $76(17.2)$ & $41(11.4)$ & $24(7.0)$ & \\
\hline Graduated high school & 785 (35.3) & $268(38.3)$ & $145(32.7)$ & $110(30.6)$ & $98(28.7)$ & \\
\hline Graduated college or more & $906(40.8)$ & $308(43.9)$ & $150(33.8)$ & $151(42.0)$ & $198(58.1)$ & \\
\hline \multicolumn{7}{|l|}{ Smoking } \\
\hline Never smoker & $1,202(53.9)$ & $478(59.1)$ & $240(46.8)$ & $183(44.9)$ & $228(62.6)$ & \multirow[t]{3}{*}{$<0.001^{\dagger}$} \\
\hline Current smoker & $349(15.7)$ & $115(14.2)$ & $84(16.4)$ & $71(17.4)$ & 55 (15.1) & \\
\hline Former smoker & $677(30.4)$ & $216(26.7)$ & $189(36.8)$ & $154(37.7)$ & $81(22.3)$ & \\
\hline \multicolumn{7}{|l|}{ Comorbid disease } \\
\hline Coronary artery disease & $118(5.3)$ & $14(1.7)$ & $60(11.6)$ & $32(7.8)$ & $2(0.5)$ & $<0.001^{\dagger}$ \\
\hline Peripheral vascular disease & $78(3.5)$ & $13(1.6)$ & $32(6.2)$ & $20(4.9)$ & $1(0.3)$ & $<0.001^{\dagger}$ \\
\hline Cerebrovascular disease & $135(6.0)$ & $19(2.3)$ & $51(9.8)$ & $38(9.3)$ & $21(5.8)$ & $<0.001^{\dagger}$ \\
\hline Diabetes & $755(33.7)$ & $70(8.6)$ & $519(100.0)$ & $72(17.6)$ & $12(3.3)$ & $<0.001^{\dagger}$ \\
\hline Hypertension & $2,150(96.1)$ & 785 (96.9) & $513(98.8)$ & $407(99.5)$ & $314(86.3)$ & $<0.001^{\dagger}$ \\
\hline Congestive heart failure & $34(1.5)$ & $7(0.9)$ & $12(2.3)$ & $10(2.4)$ & $2(0.5)$ & $0.025^{\ddagger}$ \\
\hline Arrhythmia & $56(2.5)$ & $18(2.2)$ & $16(3.1)$ & $18(4.4)$ & $2(0.5)$ & $0.006^{\dagger}$ \\
\hline \multicolumn{7}{|l|}{ Age-adjusted modified CCl } \\
\hline $\operatorname{Low}(\leq 3)$ & $1,174(52.5)$ & $627(77.4)$ & $24(4.6)$ & $153(37.4)$ & $317(87.1)$ & \multirow[t]{4}{*}{$<0.001^{\dagger}$} \\
\hline Moderate (4-5) & $652(29.1)$ & 155 (19.1) & 204 (39.3) & $193(47.2)$ & $45(12.4)$ & \\
\hline High (6-7) & $347(15.5)$ & $25(3.1)$ & $242(46.6)$ & $56(13.7)$ & $2(0.5)$ & \\
\hline Very high (8-9) & $65(2.9)$ & $3(0.4)$ & $49(9.4)$ & $7(1.7)$ & $0(0.0)$ & \\
\hline BMI $\left(\mathrm{kg} / \mathrm{m}^{2}\right)$ & $24.6 \pm 3.4$ & $24.2 \pm 3.3$ & $25.2 \pm 3.2$ & $25.1 \pm 3.5$ & $23.5 \pm 3$ & $<0.001^{*}$ \\
\hline \multicolumn{7}{|l|}{ BP variables } \\
\hline Systolic BP (mmHg) & $127.8 \pm 16.2$ & $123.4 \pm 14.2$ & $134.3 \pm 18.5$ & $127.8 \pm 15.9$ & $128.3 \pm 13.3$ & $<0.001^{*}$ \\
\hline Diastolic BP (mmHg) & $77 \pm 11.1$ & $75.6 \pm 10.1$ & $75.7 \pm 11.7$ & $77.7 \pm 11.5$ & $81 \pm 10.4$ & $<0.001^{*}$ \\
\hline Pulse pressure (mmHg) & $50.8 \pm 12.2$ & $47.8 \pm 10.6$ & $58.5 \pm 13.4$ & $50.1 \pm 11.3$ & $47.3 \pm 9.8$ & $<0.001^{*}$ \\
\hline $\operatorname{eGFR}\left(\mathrm{mL} / \mathrm{min} / 1.73 \mathrm{~m}^{2}\right)$ & $53.1 \pm 30.7$ & $60.1 \pm 31.4$ & $36.6 \pm 21.9$ & $42.3 \pm 21.7$ & $72.9 \pm 32.9$ & $<0.001^{*}$ \\
\hline Urine protein/24 hr (mg/day) & $1,353 \pm 2,139$ & $1,292 \pm 1,524$ & $2,664 \pm 3,168$ & $766 \pm 1,133$ & $178 \pm 256$ & $<0.001 *$ \\
\hline ACEI or ARB therapy & $1,907(85.4)$ & $726(89.7)$ & $448(86.8)$ & $333(81.6)$ & $285(78.3)$ & $<0.001^{\dagger}$ \\
\hline \multicolumn{7}{|l|}{ CKD stage } \\
\hline Stage G1 & $265(11.8)$ & $131(16.2)$ & $13(2.5)$ & $11(2.7)$ & $94(25.8)$ & \multirow[t]{6}{*}{$<0.001^{\dagger}$} \\
\hline Stage G2 & $419(18.7)$ & $180(22.2)$ & $44(8.5)$ & $48(11.7)$ & $118(32.4)$ & \\
\hline Stage G3a & $403(18.0)$ & $163(20.1)$ & $72(13.9)$ & $95(23.2)$ & $52(14.3)$ & \\
\hline Stage G3b & $484(21.6)$ & $158(19.5)$ & $131(25.2)$ & $112(27.4)$ & $45(12.4)$ & \\
\hline Stage G4 & $522(23.3)$ & $138(17.0)$ & $198(38.2)$ & $118(28.9)$ & $42(11.5)$ & \\
\hline Stage G5 (predialysis) & $145(6.5)$ & $40(4.9)$ & $61(11.8)$ & $25(6.1)$ & $13(3.6)$ & \\
\hline
\end{tabular}

Data are presented as mean \pm standard deviation or number $(\%)$.

ACEI, angiotensin converting enzyme inhibitor; ARB, angiotensin receptor blocker; BMI, body mass index; BP, blood pressure; CCl, Charlson comorbidity index; CKD, chronic kidney disease; DN, diabetic nephropathy; eGFR, estimated glomerular filtration rate; G, grade; GN, glomerulonephritis; HTN, hypertensive nephropathy; KNOW-CKD, KoreaN Cohort Study for Outcomes in Patients With Chronic Kidney Disease; PKD, polycystic kidney disease.

$* P$ value evaluated by Kruskal-Wallis test; ${ }^{\dagger} P$ value evaluated by chi-square test; ${ }^{\ddagger} P$ value evaluated by Fisher's exact test. 
Table 3. Incidences of main outcomes from KNOW-CKD participants as of March 2019

\begin{tabular}{|c|c|c|c|c|c|c|c|}
\hline \multirow{3}{*}{$\begin{array}{c}\text { Outcome } \\
\text { events }\end{array}$} & \multicolumn{6}{|c|}{ Event rate per 1,000 person years } & \multirow{3}{*}{$P$} \\
\hline & \multicolumn{2}{|c|}{ Total Cohort } & \multicolumn{2}{|c|}{ Diabetes } & \multicolumn{2}{|c|}{ Non-diabetes } & \\
\hline & Event number & Incidence & Event number & Incidence & Event number & Incidence & \\
\hline ESRD & 533 & 57.0 & 262 & 98.7 & 271 & 40.5 & $<0.001$ \\
\hline ESRD or eGFR halving & 662 & 73.8 & 314 & 125.1 & 348 & 53.9 & $<0.001$ \\
\hline Any CV disease & 165 & 16.2 & 88 & 28.1 & 77 & 10.9 & $<0.001$ \\
\hline Coronary event & 76 & 7.4 & 48 & 15.3 & 28 & 4.0 & $<0.001$ \\
\hline $\mathrm{CHF}$ & 9 & 0.9 & 5 & 1.6 & 4 & 0.6 & 0.121 \\
\hline Ischemic stroke & 26 & 2.5 & 17 & 5.4 & 9 & 1.3 & $<0.001$ \\
\hline Cerebral hemorrhage & 11 & 1.1 & 5 & 1.6 & 6 & 0.8 & 0.295 \\
\hline Death & 107 & 10.1 & 62 & 18.6 & 45 & 6.2 & $<0.001$ \\
\hline
\end{tabular}

CHF, congestive heart failure; CV, cardiovascular; eGFR, estimated glomerular filtration rate; ESRD, end-stage renal disease; KNOW-CKD, KoreaN Cohort Study for Outcomes in Patients With Chronic Kidney Disease.

$P$ value from Poisson regression model in the incidence comparisons of diabetes vs. non-diabetes.

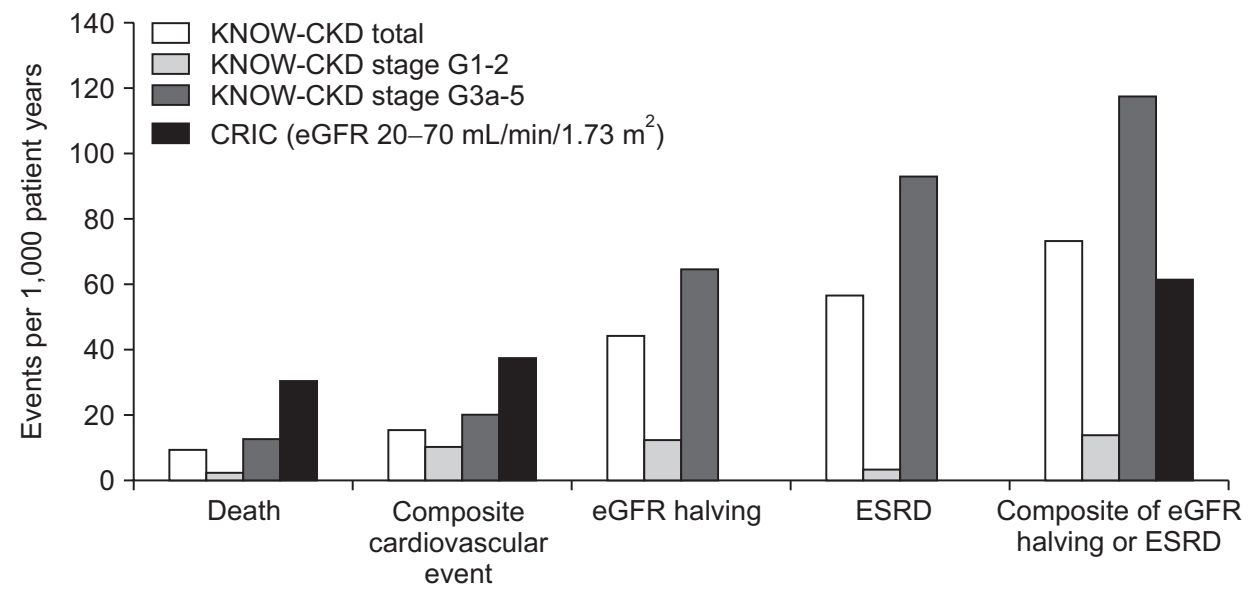

Figure 1. Incidences of major outcomes of KoreaN Cohort Study for Outcomes in Patients With Chronic Kidney Disease (KNOW-CKD) and comparison with the Chronic Renal Insufficiency Cohort (CRIC) cohort. CRIC comprises CKD subjects with estimated glomerular filtration rate (eGFR) between 20 to 70 $\mathrm{mL} / \mathrm{min} / 1.73 \mathrm{~m}^{2}$. Therefore, it is more relevant to compare CRIC and KNOW-CKD at stages G3a-5, rather than total KNOWCKD subjects.

ESRD, end-stage renal disease. a 2.2 -fold (95\% CI, 1.38-3.46) and a 2.0 -fold (95\% CI, 1.33-2.98) higher risk of CKD progression, respectively. This association was particularly evident in patients who had decreased kidney function and proteinuria [15].

\section{Cardiovascular disease profile at study entry}

Since CKD is associated with heavy CVD burden, thorough CV evaluations including echocardiography, coronary computed tomography, and pulse wave velocity (PWV) measurements were conducted at study entry and regularly thereafter. The overall prevalence of comorbid CVD at study entry was $14.4 \%$ in all study participants and was the highest in the DN subgroup followed by the HTN subgroup. The DN subgroup had more adverse $\mathrm{CV}$ risk profiles (higher systolic blood pressure (BP) and higher levels of cardiac troponin $\mathrm{T}$, left ventricular mass index, coronary calcium score, and brachial-ankle PWV) than the other subgroups. These CV risk profiles were all inversely correlated with eGFR [16]. Metabolic acidosis defined as a serum bicarbonate level of $<22 \mathrm{mmol} / \mathrm{L}$ was independently associated with arterial stiffness [17]. Vigorous investigation to search for the risk factors and biomarkers related to CVD is under way.

\section{Coronary artery calcification}

CAC is a well-known predictor of CV events and high mortality in the general population [18] and in patients with CKD $[19,20]$. The KNOW-CKD investigated the clinical and biochemical factors related to CAC. There was a gradual and independent association between low eGFR and CAC at the study entry in the KNOW-CKD subjects [21]. In view of arterial stiffness measured by PWV, the 
subjects with higher baPWV (brachial-ankle PWV) exhibited higher prevalence of CAC, suggesting that increased arterial stiffness is independently associated with CAC [22].

\section{Anemia}

Anemia is a well-known complication in CKD and is associated with the progression of CKD, poor QOL, and increase in morbidity and mortality. Overall prevalence of anemia in the KNOW-CKD cohort was $45.0 \%$, and DN as a cause, advanced CKD stages, BMI, smoking, leukocyte count, serum albumin, iron markers, calcium, and phosphorus concentration were identified as independent risk factors for anemia [23].

Recently, hepcidin was found to be a key molecule mediating iron metabolism and transport. Binding of hepcidin to ferroportin, an iron-exporting transcellular channel, induces the internalization and degradation of ferroportin and disturbs iron efflux from cells to plasma, ultimately lowering serum iron levels and sequestering iron in iron-storage sites [24]. The KNOW-CKD showed from a large number of CKD patients that lower eGFR is associated with higher serum hepcidin levels, particularly in advanced CKD. Iron supplementation was positively correlated with serum hepcidin levels, especially in patients with early CKD. The higher hepcidin level in erythropoiesis-stimulating agents (ESA) users, as compared with non-users, suggests that hepcidin is a key peptide involved in ESA resistance, suggesting a possible role of hepcidin modulation in anemia treatment [25]. Indices of iron profiles were analyzed in relation to anemia in CKD. Transferrin saturation and hepcidin were significantly associated with anemia status, whereas serum ferritin was not. Hepcidin was particularly associated with severe anemia in advanced CKD stages [26], suggesting a possible role of hepcidin as a predictive marker of anemia in CKD.

\section{Mineral bone disease of CKD}

In KNOW-CKD study patients, serum vitamin D levels began to decline in the early stages of CKD before significant elevations in fibroblast growth factor 23 (FGF23) and intact parathyroid hormone (iPTH). Moreover, FGF23 elevation was observed concurrently with increased iPTH with declining eGFR [27], in contrast to Western populations. There is a stepwise decrease in serum Klotho concentration with the decline of eGFR [28]. The prevalence of osteoporosis was higher in advanced CKD patients [27]. Also, increased vascular calcification scores are noted in both early and advanced stages of CKD, similar to that of Western populations [27].

In female patients with CKD, increased serum osteoprotegerin (OPG) levels were independently associated with decreased bone mineral density in the lumbar spine and total hip and with increased risk of osteoporosis. Therefore, measurement of serum OPG concentration may be useful as a predictor for determining bone loss in CKD patients [29].

Moreover, there may be a crosslink between the markers of bone mineral metabolism and those of anemia. The KNOW-CKD showed that elevated serum phosphorus and iPTH are associated with an increase in hepcidin, while elevated vitamin D levels are associated with a decrease in serum hepcidin levels after adjustment [30]. High FGF23 was associated with anemia at baseline and development of anemia during follow-up [31], which is consistent with the observation from the CRIC cohort [32]. Taken together, these findings suggest that correcting mineral metabolic derangement may be a possible therapeutic approach in treating anemia in CKD.

\section{Nutrition and OQOL in CKD}

Malnutrition or protein-energy wasting (PEW) in CKD is prevalent with decreasing renal function and is a strong indicator of adverse outcomes. Multiple mechanisms are involved in nutritional problems in CKD patients. Insufficient food intake caused by uremia-induced anorexia alone does not account for the unfavorable nutritional changes. There are additional contributing factors, such as chronic inflammation, acidosis, multiple hormonal disturbances, comorbidities, and physical inactivity [33]. The prevalence of PEW in the KNOW-CKD study was about $9.0 \%$ [34] according to the International Society of Renal Nutrition and Metabolism (ISRNM) criteria [35] and tended to increase with advanced stage in pre-dialysis CKD. In the multivariate logistic regression model, PEW was independently associated with eGFR, total $\mathrm{CO}_{2}$, physical activity, diabetes, and high sensitivity C-reactive protein (hs-CRP) [34]. 
Oh, et al. The KNOW-CKD Study: Major findings

Health-related quality of life (HRQOL) is a concept in QOL that focuses on the effects of illness, and specifically on the impact that treatment may have on QOL. It is generally accepted that HRQOL represents the positive and negative aspects of patients' symptoms, including emotional, social, and cognitive functions, disease burden, and treatment side effects. Recently, HRQOL has been accepted as an important health outcome. Patients with advanced CKD have multiple, complex comorbid conditions that can produce poor HRQOL [36]. The KNOWCKD study acquires information on HRQOL, SES such as occupation, academic year, monthly income, sleep quality and quantity, and health-related habits through patient-reported questionnaires at the study entry and regularly thereafter. There was a significant association between serum vitamin $D$ levels and kidney disease component summary (KDCS) and mental component summary (MCS) scores in pre-dialysis CKD patients [37]. Sleep duration was also correlated with HRQOL in patients with CKD. Too long or short sleep durations were associated with poor HRQOL [38]. WC is a better indicator of poor physical HRQOL than BMI. The association between WC and physical HRQOL is modified by age, sex, eGFR, and comorbidities such as diabetes and CVD [39].

The second phase of the KNOW-CKD study (KNOWCKD Phase II), which is scheduled to recruit 1,500 subjects between 2019 and 2021, will emphasize nutritional assessment and evaluation of QOL and cognitive function change. It is strongly anticipated that the KNOWCKD Phase II will provide us with robust medical and clinical knowledge on the nutritional issues and QOL in CKD patients.

\section{CKD progression}

The KNOW-CKD defined the renal end-point as either eGFR halving or ESRD occurrence. The composite renal event was defined as one of the two renal events above. General risk factors of renal composite end-point from the KNOW-CKD are depicted in Table 4. As a surrogate of renal progression, eGFR slope was calculated based on the mixed model.

Elucidating and modulating the major risk factors of CKD to delay progression are the key aims of the KNOWCKD study. Regarding the association between obesity
Table 4. Risk factors for renal composite end-points

\begin{tabular}{|c|c|c|c|}
\hline & $\begin{array}{l}\text { Number of } \\
\text { events (\%) }\end{array}$ & $\begin{array}{c}\text { Hazard } \\
\text { Ratio }\end{array}$ & $95 \% \mathrm{Cl}$ \\
\hline \multicolumn{4}{|l|}{ Age } \\
\hline $20-39$ & 81 (25.6) & 1 & \\
\hline $40-49$ & 131 (29.7) & 0.73 & $0.55-0.96$ \\
\hline $50-59$ & 207 (31.7) & 0.52 & $0.40-0.68$ \\
\hline $60-69$ & $182(32.3)$ & 0.41 & $0.31-0.54$ \\
\hline$\geq 70$ & 61 (29.6) & 0.34 & $0.24-0.48$ \\
\hline \multicolumn{4}{|l|}{ Gender } \\
\hline Female & 266 (31.6) & 1 & \\
\hline Male & 396 (29.6) & 1.14 & $0.97-1.33$ \\
\hline \multicolumn{4}{|l|}{ DM } \\
\hline Non-DM & $348(24.0)$ & 1 & \\
\hline DM & 314 (43.1) & 1.95 & $1.66-2.30$ \\
\hline \multicolumn{4}{|l|}{ CKD stage } \\
\hline G1 & $12(3.4)$ & 1 & \\
\hline G2 & 48 (11.5) & 4.10 & $2.17-7.74$ \\
\hline G3a & 66 (18.4) & 7.46 & $4.00-13.89$ \\
\hline G3b & 149 (32.4) & 14.04 & $7.72-25.51$ \\
\hline G4 & 275 (59.5) & 41.19 & $22.85-74.27$ \\
\hline G5 & $112(84.8)$ & 145.24 & $79.12-266.64$ \\
\hline
\end{tabular}

Multivariable analysis by Cox proportional hazards model.

$\mathrm{Cl}$, confidence interval; CKD, chronic kidney disease; DM, diabetes mellitus; G, grade.

and CKD progression, there have been conflicting results. Here, we defined metabolic abnormality as the presence of three or more of the following five components: hypertension, fasting glucose level $\geq 126 \mathrm{mg} / \mathrm{dL}$ or the presence of type 2 diabetes, triglyceride level $>150 \mathrm{mg} / \mathrm{dL}$ or use of lipid-lowering drugs, high-density lipoprotein cholesterol level $\leq 40 \mathrm{mg} / \mathrm{dL}$ in men and $\leq 50 \mathrm{mg} / \mathrm{dL}$ in women, and hs-CRP level $>1 \mathrm{mg} / \mathrm{L}$. Obesity was defined as BMI $\geq 25 \mathrm{~kg} / \mathrm{m}^{2}$. The KNOW-CKD showed that both metabolic abnormalities and obesity are associated with a significantly increased risk for CKD progression. Notably, obese patients without metabolic abnormalities also have an elevated risk for CKD progression (Fig. 2) [40]. Another study investigated the effect of dietary salt intake on CKD progression using 24-hour urinary Na excretion as a proxy [41]. Here, it was shown that, compared with the reference group (urinary Na excretion 104.2-145.1 mEq/ day), the highest quartile group was associated with 1.8 times higher risk of composite renal outcome (urinary $\mathrm{Na}$ excretion $\geq 192.9 \mathrm{mEq} /$ day, $P=0.015$ ) in a multivariable hazards model (Fig. 3) [41]. Arterial stiffness measured by 


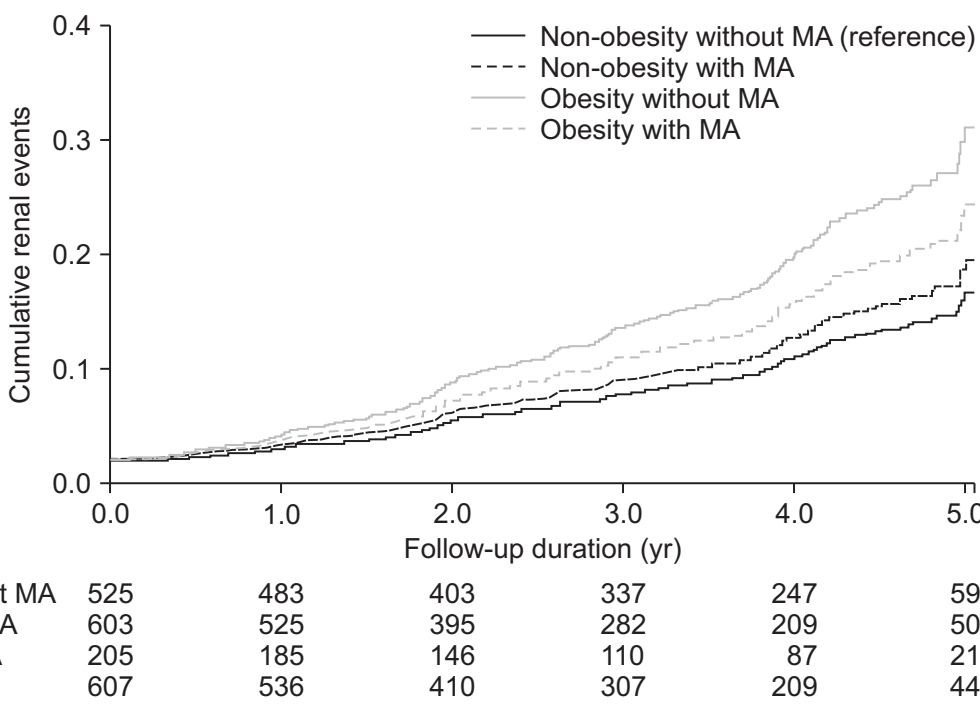

Figure 2. Adjusted Kaplan-Meier curve for cumulative renal events according

Number at risk Non-obesity without MA 525 Non-obesity with MA Obesity without MA Obesity with MA
607

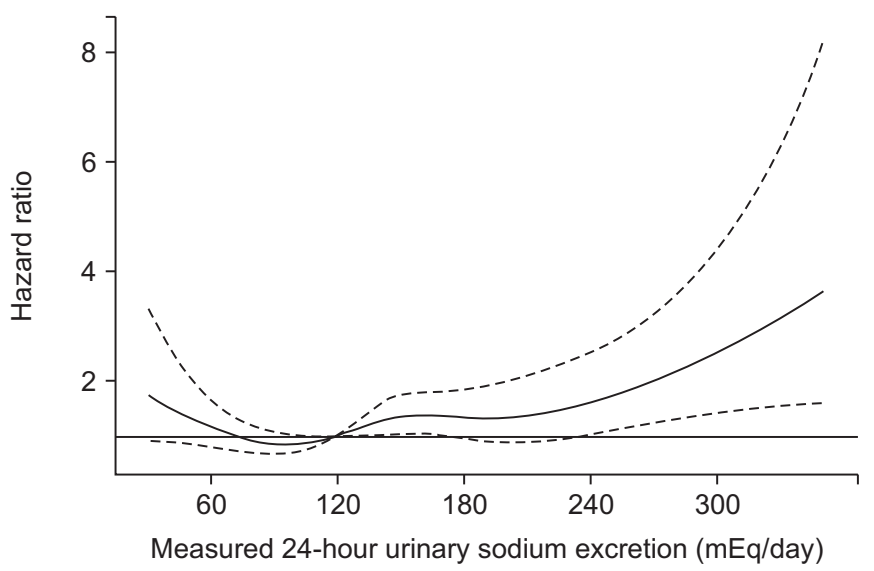

Figure 3. Association of measured 24-hour urinary sodium excretion with hazard ratio of chronic kidney disease progression in fully-adjusted model [41]. Note the relatively linear relationship between measured 24-hour urinary sodium excretion and risk of composite renal outcomes at the reference of urinary sodium excretion $\geq 120 \mathrm{mEq} /$ day.

PWV was also shown to be a risk factor for renal outcome. High baPWV, if accompanied by a high heart-to-femoral PWV (hfPWV), was shown to be associated with rapid eGFR decline, suggesting that central and peripheral PWVs may simultaneously affect rapid eGFR decline [42].

Dietary protein restriction may be beneficial to CKD patients by delaying CKD progression. However, it may be harmful to patients at risk of PEW. Therefore, dietary protein restriction may be a double-edged sword for CKD patients. The KNOW-CKD analyzed associations between dietary protein intake (DPI) assessed by 24 -hour urine nitrogen excretion [43], PEW, and renal outcome. Low
DPI was found to be associated with PEW, which may attenuate the potential benefits of a low-protein diet on CKD progression. Therefore, caution about the risk of PEW particularly needs to be expressed to patients [44].

To date, several biochemical markers shown to be independently associated with CKD progression by the KNOW-CKD study are hyperuricemia [45], low urine osmolality [46], low urine chloride [47], and low urine potassium [48] levels.

\section{CV outcome and death in KNOW-CKD}

As mentioned above, among 2,238 subjects, there were 107 deaths and 165 fatal or non-fatal CV events as of March 31, 2019. Compared with the incidences of renal end-points, the incidences of CV outcomes and death were relatively low, as shown in Table 3. Observational studies from Western CKD populations reported that patients with CKD have a higher risk of death than risk of progression to ESRD [49]. However, in our Korean CKD population, incidences of any CV event (16.2 per 1,000 patient-years) or all-cause death (10.1 per 1,000 patientyears) were much lower than those of ESRD, which were 57 per 1,000 patient-years. This is similar to the report from Japanese CKD subjects [50] and was also shown in a recent international collaborative study comparing the outcomes of various CKD cohorts [51]. Investigation on the risk factors of adverse CV outcome or death will be conducted when a significant number of end-points is reached to ensure statistical power. 

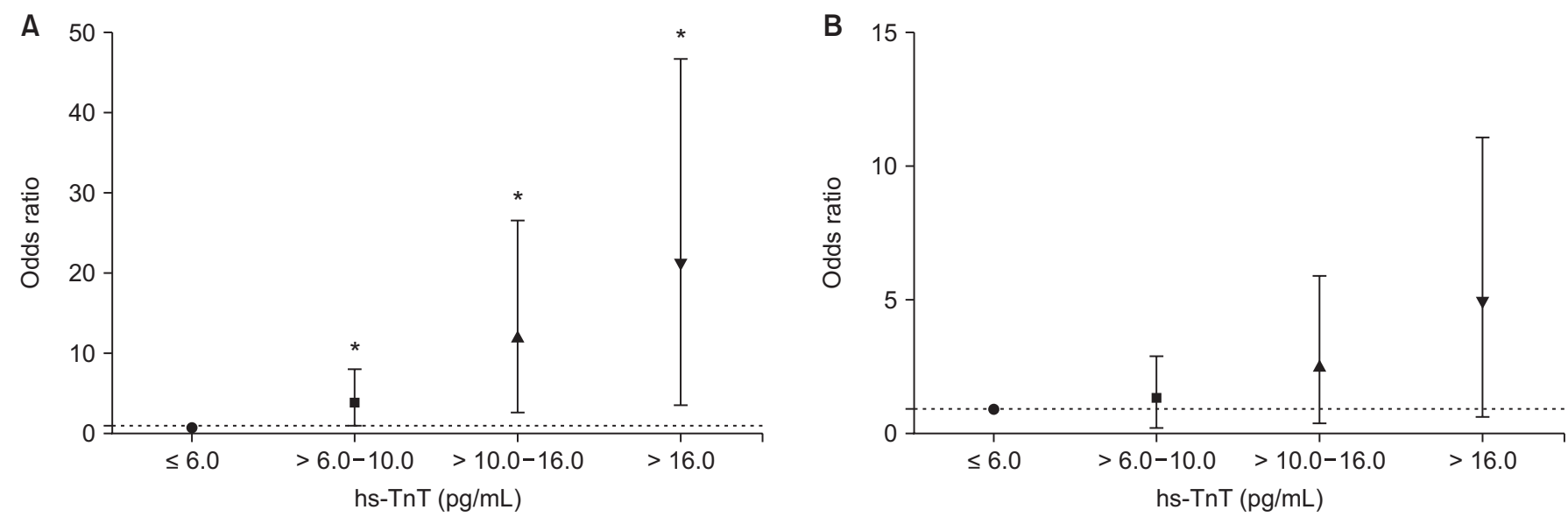

Figure 4. The association between high-sensitivity troponin T (hs-TnT) and longitudinal echocardiographic changes [61]. (A) Left ventricular hypertrophy: adjusted to age, sex, mean arterial pressure, diabetes mellitus (DM), hypertension (HTN), coronary artery disease, chronic kidney disease (CKD) stage, body mass index (BMI), high density lipoprotein cholesterol (HDL-C), triglyceride (TG), hemoglobin. (B) Diastolic dysfunction: adjusted to age, sex, DM, HTN, CKD stage, BMI, HDL-C, TG, C-reactive protein, smoking history.

\section{Biomarker discovery in CKD}

Biomarkers are molecules that are objectively measured and evaluated as an indicator of normal biological process, pathologic process, or pharmacologic response. Recent advances in molecular biology, functional genomics, and proteomics have elucidated many candidate biomarkers in acute kidney injury and CKD, such as neutrophil gelatinase-associated lipocalin, kidney injury molecule-1, and liver-type fatty acid-binding protein. The KNOW-CKD has found several biomarker candidate molecules that can predict major adverse outcomes of CKD. Candidate biomarkers of CKD outcomes are cystatin C [21], albuminuria [52], adiponectin [53-58], FGF23 $[31,59]$, high density lipoprotein cholesterol [60], troponin T [61] (Fig. 4), hepcidin [26,30], Klotho [28,62], OPG [63], and various urinary markers $[46,47,64-66]$. The main results of the biomarker studies are described in Table 5 [28,53-66].

\section{International collaborations for CKD study}

The incidence, prevalence, and clinical outcomes of CKD/ESRD vary depending on country and ethnicity $[67,68]$. The KNOW-CKD has been actively participating in the International Network of CKD Cohort Studies (iNET-CKD) [69] in pursuit of the factors explaining such differences through inter-ethnic and international comparison and meta-analysis.
In one recent meta-analysis [70], the iNET-CKD reported crude prevalence of uncontrolled BP among 17 cohort studies, including 34,602 individuals with eGFR $<60$ $\mathrm{mL} / \mathrm{min} / 1.73 \mathrm{~m}^{2}$ and treated hypertension adjusted for potential confounders. Crude prevalence of $\mathrm{BP} \geq 140 / 90$ mmHg ranged from $28 \%$ to $61 \%$. Adjusted prevalence ratios indicated poorer hypertension control in cohorts from European countries, India, and Uruguay, and better control in North America and high-income Asian countries including Korea and Japan. Particularly, the KNOWCKD exhibited the lowest prevalence of uncontrolled hypertension (27.3\%) among the 17 participating CKD cohorts.

Another international meta-analysis study compared the longitudinal outcomes of CKD across eight iNET-CKD cohorts [51]. The risks of CKD progression, ESRD, death, and CVD vary across countries even after adjusting for age, sex, comorbidities, and baseline laboratory results. The KNOW-CKD cohort population, as compared to the other seven cohorts in Europe, Japan, North America, and South America, exhibited relatively lower incidence of all-cause mortality, while exhibiting higher incidences of CKD progression and ESRD, after adjustment (Fig. 5).

Taken together, the findings from the two international comparison studies above warrant a search for the specific genetic, socio-economic, and cultural factors in the Korean CKD population that explain this difference. 
Table 5. Summary of biomarker discovery studies in KNOW-CKD

\begin{tabular}{|c|c|c|c|}
\hline Time frame & Biomarker & Outcome & Remarks \\
\hline $\mathrm{C}$ & Adiponectin & MS & Hypoadiponectinemia is independently associated with the metabolic syndrome in CKD [53]. \\
\hline $\mathrm{C}$ & Adiponectin & PEW & $\begin{array}{l}\text { High serum adiponectin is independently associated with PEW. Among PEW indicators, } \\
\text { serum adiponectin is closely associated with urine creatinine excretion as an indirect } \\
\text { measure of muscle mass [54]. }\end{array}$ \\
\hline $\mathrm{C}$ & Adiponectin & $\begin{array}{l}\text { Albuminuria and } \\
\text { renal function }\end{array}$ & $\begin{array}{l}\text { Serum adiponectin is higher in patients with increasing albuminuria, and these levels are } \\
\text { associated with albuminuria, renal insufficiency and lipid profiles [55]. }\end{array}$ \\
\hline $\mathrm{C}$ & Adiponectin & hfPWV & $\begin{array}{l}\text { The independent and significant correlation of serum adiponectin concentration with } \\
\text { hfPWV in CKD implicates adiponectin in CKD-associated aortic stiffness [56]. }\end{array}$ \\
\hline $\mathrm{C}, \mathrm{L}$ & Adiponectin & Anemia & $\begin{array}{l}\text { A high serum adiponectin level is independently associated with a low hemoglobin level } \\
\text { and predicts the development of anemia in patients with CKD [57]. }\end{array}$ \\
\hline $\mathrm{L}$ & Adiponectin & Renal event & $\begin{array}{l}\text { Serum adiponectin may be a biomarker of renal dysfunction rather than a true risk factor } \\
\text { in CKD progression [58]. }\end{array}$ \\
\hline $\mathrm{C}$ & FGF23 & CAC & $\begin{array}{l}\text { High serum FGF23 was associated with CAC in CKD patients with high adiponectin, but not } \\
\text { in those with low adiponectin [59]. }\end{array}$ \\
\hline $\mathrm{L}$ & HDL cholesterol & Renal event & $\begin{array}{l}\text { A U-shaped association was observed between serum HDL-C levels and adverse renal } \\
\text { outcomes [60]. }\end{array}$ \\
\hline $\mathrm{C}, \mathrm{L}$ & hs-TnT & $\begin{array}{l}\text { LV structure and } \\
\text { function, new LVH }\end{array}$ & $\begin{array}{l}\text { hs-TnT is strongly associated with alterations of LV structure and diastolic dysfunction for } \\
\text { both eGFR strata. Baseline hs-TnT levels are predictive of new LVH on follow-up [61]. }\end{array}$ \\
\hline $\mathrm{C}$ & Klotho & LV mass index & Serum Klotho is an independent biomarker of left ventricular mass index [28]. \\
\hline $\mathrm{C}$ & Klotho & MS & $\begin{array}{l}\text { MS is prevalent in CKD. Serum Klotho is inversely associated with the presence of MS in } \\
\text { patients with CKD [62]. }\end{array}$ \\
\hline $\mathrm{C}$ & OPG & baPWV & $\begin{array}{l}\text { Non-traditional, kidney-related cardiovascular risk factors in addition to traditional } \\
\text { cardiovascular risk factors are related to serum level of OPG in CKD. Serum OPG level is } \\
\text { significantly related to baPWV [63]. }\end{array}$ \\
\hline $\mathrm{C}$ & $\begin{array}{l}\text { Urine creatinine } \\
\text { excretion }\end{array}$ & PWV & $\begin{array}{l}\text { 24-hour urine creatinine is a marker of muscle mass. Low creatinine excretion is } \\
\text { associated with high PWV, suggesting a relationship between decreased muscle mass } \\
\text { and arterial stiffness [64]. }\end{array}$ \\
\hline $\mathrm{L}$ & Urine $\mathrm{Na} / \mathrm{K}$ ratio & Renal event & $\begin{array}{l}\text { The } \mathrm{Na} / \mathrm{K} \text { ratio in urine is an index of dietary sodium and potassium intake. The higher } \mathrm{Na} / \\
\mathrm{K} \text { ratio in 24-hour urine collection is associated with higher rate of } \mathrm{CKD} \text { progression [65]. }\end{array}$ \\
\hline $\mathrm{C}, \mathrm{L}$ & Urine AGT & $\begin{array}{l}\text { Urinary K excretion, } \\
\text { renal event }\end{array}$ & $\begin{array}{l}\text { High urinary AGT level is associated with increased urinary K excretion and renal outcome } \\
\text { in patients with PKD [66]. }\end{array}$ \\
\hline
\end{tabular}

AGT, angiotensinogen; baPWV, brachial-to-ankle pulse wave velocity; C, cross-sectional; CAC, coronary artery calcification; CKD, chronic kidney disease; FGF23, fibroblast growth factor 23; HDL-C, high density lipoprotein cholesterol; hfPWV, heart-to-femoral pulse wave velocity; hs-TnT, high sensitivity troponin T; KNOWCKD, KoreaN Cohort Study for Outcomes in Patients With Chronic Kidney Disease; L, longitudinal; LV, left ventricle; LVH, left ventricular hypertrophy; MS, metabolic syndrome; OPG, osteoprotegerin; PEW, protein-energy wasting; PKD, polycystic kidney disease.

\section{KNOW-CKD Phase II}

The KNOW-CKD has recently launched Phase II enrollment for 2019 through 2021 (Table 6). The aims of the KNOW-CKD Phase II study are as follows: 1 ) to enlarge the total pool of CKD patients in the cohort, particularly those with eGFR $<60 \mathrm{~mL} / \mathrm{min} / 1.73 \mathrm{~m}^{2}$ and high risks of renal progression 2) to focus more on DN and HTN, and 3) to emphasize the health habits of the CKD patients by obtaining more information on dietary patterns, nutritional status, and cognitive functions. It is expected that the KNOW-CKD Phase II will not only reinforce the sta- tistical power of the KNOW-CKD Phase I but also provide robust scientific evidence on the significance of health habits and dietary modification on the CKD outcomes.

\section{Summary and conclusion}

As the nation's largest CKD cohort, the KNOW-CKD has relentlessly pursued answers to the basic questions on clinical courses and elucidation of the risk factors for adverse outcomes from the Korean CKD population. Particularly, among various aspects of CKD, greater emphases were placed upon socio-economics, nutrition and 
A Incidences of all cause death

\begin{tabular}{|c|c|c|c|c|c|c|}
\hline \multicolumn{3}{|c|}{ Study } & \multicolumn{2}{|c|}{$\begin{array}{r}\text { Incidence rates }(95 \% \mathrm{Cl}) \\
\text { (events/1,000 person-years) }\end{array}$} & \multirow{2}{*}{$\begin{array}{c}\text { Number of } \\
\text { events } \\
105\end{array}$} & \multirow{2}{*}{$\begin{array}{c}\begin{array}{c}\text { Person- } \\
\text { years }\end{array} \\
\mathbf{2 , 1 6 6}\end{array}$} \\
\hline * $\because$ & CKD-QLD (AUS) & $\multimap$ & & $48(40-57)$ & & \\
\hline $\begin{array}{ll}\text { NV/ } \\
\mathbb{Z N}\end{array}$ & RIISC (GBR) & $\longrightarrow$ & & $48(39-57)$ & 130 & 2,698 \\
\hline ״ & CRIC (USA) & $\bullet$ & & $43(12-75)$ & 990 & 22,808 \\
\hline "I") & KNOW-CKD (KOR) & & & $7(4-10)$ & 26 & 3,705 \\
\hline 4 & CAN-PREDDICT (CAN) & $\multimap$ & & $38(30-46)$ & 301 & 7,912 \\
\hline 絭 & NRHP (URY) & $\vdash$ & & $45(44-47)$ & 2,878 & 63,842 \\
\hline 0 & CKD-JAC (JPN) & & & $10(6-15)$ & 82 & 7,946 \\
\hline & $\begin{array}{l}10 \\
\text { Incide } \\
\text { (ever }\end{array}$ & $\begin{array}{l}30 \quad 50 \\
\text { ce rates of al } \\
/ 1,000 \text { persor }\end{array}$ & $\begin{array}{l}70 \quad 90 \\
\text { cause mortality } \\
\text { years) }\end{array}$ & $\begin{array}{r}\text { Cochran's } \\
I^{2}(95 \%\end{array}$ & $\begin{array}{l}\text { 2: } 640.27 \text { Df }(6 \\
\mathrm{Cl}): 99.1 \%(9\end{array}$ & $\begin{array}{l}p<0.001 \\
8-99.3 \%)\end{array}$ \\
\hline
\end{tabular}

B Incidences of eGFR halving or ESRD

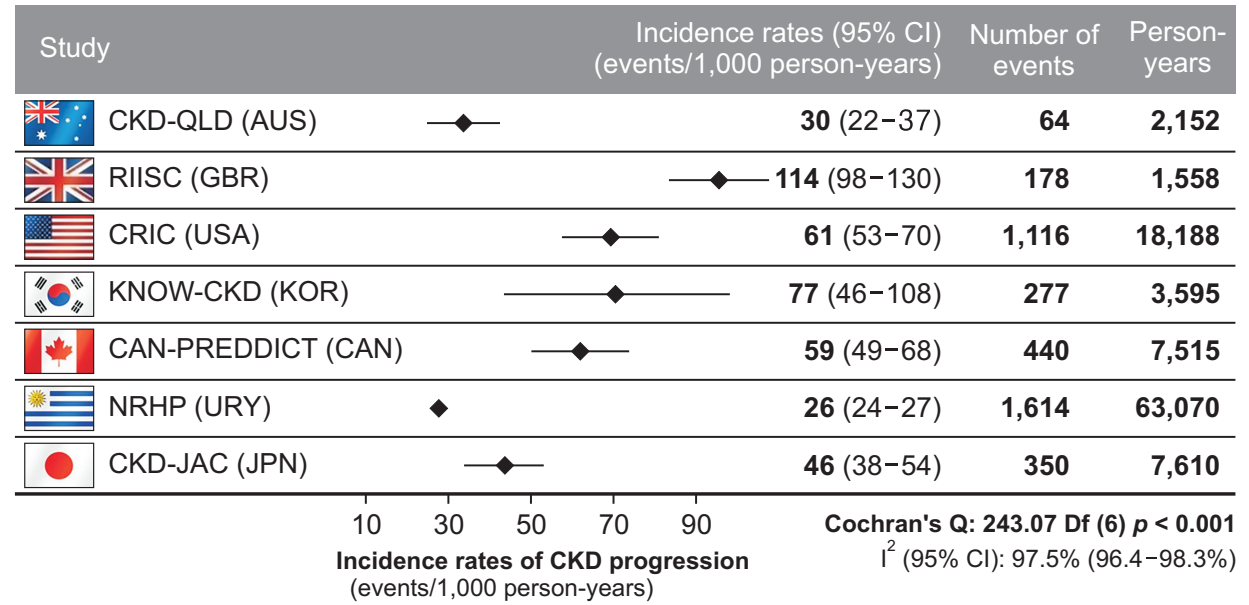

Figure 5. Adjusted incidence rates of all-cause death $(A)$ and composite of estimated glomerular filtration rate (eGFR) halving or end-stage renal disease (ESRD) (B) across the International Network of Chronic Kidney Disease studies (iNET-CKD) [51], reproduced with permission. Adjusted incidence rates were estimated through direct standardization using the total population from all study groups as the standard. Adjustments included age, sex, and eGFR at baseline. Individuals included were 18 years and older and had an eGFR assessment at baseline between 15 and $60 \mathrm{~mL} / \mathrm{min}$ per $1.73 \mathrm{~m}^{2}$. Cochran's Q and $\mathrm{I}^{2}$ statistics were estimated to assess heterogeneity in incidence rates across iNET-CKD studies.

AUS, Australia; Cl, confidence interval; CAN, Canada; CAN-PREDDICT, Canadian Study of Prediction of Death, Dialysis and Interim Cardiovascular Events; CKD-JAC, Chronic Kidney Disease Japan Cohort; CKD-QLD, Chronic Kidney Disease in Queensland; CRIC, Chronic Renal Insufficiency Study; GBR, Great Britain; JPN, Japan; KNOW-CKD, KoreaN Cohort Study for Outcomes in Patients With Chronic Kidney Disease; KOR, Republic of Korea; NRHP, National Renal Healthcare Program; RIISC, Renal Impairment in Secondary Care; URY, Uruguay; USA, United States of America.

QOL, health habits, CKD progression, CV comorbidity and outcome, anemia, mineral bone disease, biomarker discovery, and international and inter-ethnic comparison. By following the subjects for more than 10 years, the KNOW-CKD will eventually offer a prediction model for the long-term consequences of CKD such as the occurrences of ESRD, CVD, and death, thereby enabling the identification and treatment of the at-risk population that requires extra medical attention.

\section{The KNOW-CKD Investigator Group}

Patient Recruitment. Seoul National University Hospital, Curie Ahn, MD, Kook-Hwan Oh, MD (PI), Hajeong Lee, MD, Seung Seok Han, MD, Hyunjin Ryu, MD, Eunjeong Kang, MD, Minjung Kang, MD, Youngok Ko, RN, Jeongok So, RN, and Aram Lee, RN. Seoul National University Bundang Hospital, Dong Wan Chae, MD (SubPI), Yong Jin Yi MD, Hyun Jin Cho, RN and Jung Eun Oh RN. 
Table 6. Basic design and enrollment in Phase I and II of the KNOW-CKD study

\begin{tabular}{lcc}
\hline \multicolumn{1}{c}{ Characteristics } & Phase I & Phase II \\
\hline Timing of enrollment (year) & $2011-2016$ & $2019-2021$ \\
Number of participating & 9 & 13 \\
$\quad$ centers & & \\
Number enrolled & 2,238 & $1,500^{\mathrm{a}}$ \\
Age (yr) & $20-75$ & $45-79$ \\
CKD Stage or eGFR & CKD G1-G5 & $20-60$ \\
& (pre-dialysis) & $\mathrm{mL} / \mathrm{min} / 1.73 \mathrm{~m}^{2}$ \\
Diabetes & $34 \%$ & $50 \%^{\mathrm{a}}$ \\
\hline
\end{tabular}

CKD, chronic kidney disease; eGFR, estimated glomerular filtration; KNOW-CKD, KoreaN Cohort Study for Outcomes in Patients With Chronic Kidney Disease. ${ }^{\text {aplanned. }}$

Yonsei University, Severance Hospital, Kyu Hun Choi, MD (SubPI), Seung Hyeok Han, MD, Tae- Hyun Yoo, MD, and Mi Hyun Yu, RN. Kangbuk Samsung Hospital, Kyu-Beck Lee, MD and Young Youl Hyun, MD, Hyun Jung Kim, RN. The Catholic University of Korea, Seoul St. Mary's Hospital, Yong-Soo Kim, MD and Sol Ji Kim, RN. Gachon University Gil Medical Center, Wookyung Chung, MD, Ji Yong Jung, MD and Kwon Eun Jin, RN. Nowon Eulji Medical Center, Eulji University. Su Ah Sung, MD, Sung Woo Lee, MD, Hyang Ki Min, MD, and Soon Bin Kwon, RN. Chonnam National University Hospital, Soo Wan Kim, MD, Seong Kwon Ma, MD, Eun Hui Bae, MD, Chang Seong Kim, MD, Hong Sang Choi, MD, Minah Kim, MD, Tae Ryom Oh, MD, Sang Heon Suh, MD, Su Hyun Song, MD, and Se Jeong Lee, RN. Inje University, Pusan Paik Hospital, Yeong Hoon Kim, MD, Sun Woo Kang, MD, Hoseok Koo, MD, Tae Hee Kim, MD and Yun Mi Kim. MD and Young Eun Oh, MSc. Pusan National University Hospital, Eun Young Seong, MD, Sang Heon Song, MD, Miyeun Han, MD, Hyo Jin Kim, MD, Seunghee Ji, RN, National Health Insurance Service Ilsan Hospital, Tae Ik Chang, MD, Ea Wha Kang, MD, Kyoung Sook Park, MD, Aei Kyung Choi, RN. Hallym University Dongtan Sacred Heart Hospital Ja-Ryong Koo, MD, Jang-Won Seo, MD, Sun Ryoung Choi, MD, Seon Ha Baek, MD and Myung Sun Kim, RN. Seoul National University Boramae Medical Center, Yun Kyu Oh, MD (SubPI), Jeong Mi Park, RN.

Epidemiology and Biostatistics. Department of Preventive Medicine, Seoul National University College of Medicine, Byung-Joo Park, MD, Sue K. Park, MD, Choonghyun
Ahn, MD and Kyungsik Kim, BSc. School of Medicine, Inha University Department of Prevention and Management, Inha University Hospital, Joongyub Lee, MD.

Data Coordinating Center. Medical Research Collaborating Center, Seoul National University Hospital and Seoul National University College of Medicine, Jayoun Kim, PhD, Dayeon Nam, RN, Soohee Kang, MSc, Juhee Lee, MSc, and Heejung Ahn, RN. Central Laboratory. Dong Hee Seo, MD, and Soyoung Kim, MD, LabGenomics, Korea. Biobank. Korea Biobank, Korea Centers for Disease Control and Prevention, Osong, Korea. Korea Center for Disease Control and Prevention. Ok Park, Il Yoel Kim, Sung Hyun Kang, and Kyoung Hwa Kim

Details on the design, patient enrollment, investigator group, research publications, and annual report are available at the website of the KNOW-CKD study (http:// www.know-ckd.org).

\section{Conflicts of interest}

All authors have no conflicts of interest to declare.

\section{Funding}

This study was supported by the Research Program funded by the Korea Center for Disease Control and Prevention (2011E3300300, 2012E3301100, 2013E3301600, 2013E3301601, 2013E3301602, 2016E3300200, and 2019E320100).

\section{Acknowledgments}

Authors thank all the investigators and clinical research coordinators for their consistent and dedicated contribution to this long-term cohort study.

\section{Authors' contributions}

All of the authors contributed to the formation of the overall concept of the study. Minjung Kang, Eunjeong Kang, and Hyunjin Ryu drafted the manuscript. KookHwan Oh revised and edited the manuscript. Kook-Hwan Oh, Seung Hyeok Han, Tae-Hyun Yoo, Soo Wan Kim, Sue K. Park, Kyu-Beck Lee, Dong-Wan Chae, Yeong Hoon Kim, and Curie Ahn designed the main concept of the study. Curie Ahn and Kook-Hwan Oh funded the study. 


\section{References}

[1] Kang YU, Bae EH, Ma SK, Kim SW. Determinants and burden of chronic kidney disease in a high-risk population in Korea: results from a cross-sectional study. Korean J Intern Med 2016;31:920-929.

[2] Ji E, Kim YS. Prevalence of chronic kidney disease defined by using CKD-EPI equation and albumin-to-creatinine ratio in the Korean adult population. Korean J Intern Med 2016;31:1120-1130.

[3] Jin DC, Yun SR, Lee SW, et al. Lessons from 30 years' data of Korean end-stage renal disease registry, 1985-2015. Kidney Res Clin Pract 2015;34:132-139.

[4] Oh KH, Park SK, Park HC, et al. KNOW-CKD (KoreaN cohort study for Outcome in patients With Chronic Kidney Disease): design and methods. BMC Nephrol 2014;15:80.

[5] Kang E, Han M, Kim H, et al. Baseline general characteristics of the Korean Chronic Kidney Disease: report from the KoreaN Cohort Study for Outcomes in Patients With Chronic Kidney Disease (KNOW-CKD). J Korean Med Sci 2017;32:221-230.

[6] Feldman HI, Appel LJ, Chertow GM, et al. The Chronic Renal Insufficiency Cohort (CRIC) study: design and methods. J Am Soc Nephrol 2003;14(7 Suppl 2):S148-S153.

[7] Lash JP, Go AS, Appel LJ, et al. Chronic Renal Insufficiency Cohort (CRIC) study: baseline characteristics and associations with kidney function. Clin J Am Soc Nephrol 2009;4: 1302-1311.

[8] Imai E, Matsuo S, Makino H, et al. Chronic Kidney Disease Japan Cohort (CKD-JAC) study: design and methods. Hypertens Res 2008;31:1101-1107.

[9] Levey AS, Stevens LA, Schmid CH, et al. A new equation to estimate glomerular filtration rate. Ann Intern Med 2009; 150:604-612.

[10] Norton JM, Moxey-Mims MM, Eggers PW, et al. Social determinants of racial disparities in CKD. J Am Soc Nephrol 2016;27:2576-2595.

[11] Kang E, Lee J, Kim HJ, et al. The association between socioeconomic disparities and left ventricular hypertrophy in chronic kidney disease: results from the KoreaN Cohort Study for Outcomes in Patients With Chronic Kidney Disease (KNOW-CKD). BMC Nephrol 2018;19:203.

[12] Johansen KL, Young B, Kaysen GA, Chertow GM. Association of body size with outcomes among patients beginning dialysis. Am J Clin Nutr 2004;80:324-332.

[13] Kalantar-Zadeh K, Kopple JD, Kilpatrick RD, et al. Associa- tion of morbid obesity and weight change over time with cardiovascular survival in hemodialysis population. Am J Kidney Dis 2005;46:489-500.

[14] Lee MJ, Park JT, Park KS, et al. Normal body mass index with central obesity has increased risk of coronary artery calcification in Korean patients with chronic kidney disease. Kidney Int 2016;90:1368-1376.

[15] Joo YS, Koh H, Nam KH, et al. Alcohol consumption and progression of chronic kidney disease: results from the Korean Cohort Study for Outcome in Patients With Chronic Kidney Disease. Mayo Clin Proc 2020;95:293-305.

[16] Kim H, Yoo TH, Choi KH, et al. Baseline cardiovascular characteristics of adult patients with chronic kidney disease from the KoreaN Cohort Study for Outcomes in Patients With Chronic Kidney Disease (KNOW-CKD). J Korean Med Sci 2017;32:231-239.

[17] Kim HJ, Kang E, Ryu H, et al. Metabolic acidosis is associated with pulse wave velocity in chronic kidney disease: results from the KNOW-CKD Study. Sci Rep 2019;9:16139.

[18] Budoff MJ, Shaw LJ, Liu ST, et al. Long-term prognosis associated with coronary calcification: observations from a registry of 25,253 patients. J Am Coll Cardiol 2007;49:18601870.

[19] Block GA, Raggi P, Bellasi A, Kooienga L, Spiegel DM. Mortality effect of coronary calcification and phosphate binder choice in incident hemodialysis patients. Kidney Int 2007; 71:438-441.

[20] Russo D, Corrao S, Battaglia Y, et al. Progression of coronary artery calcification and cardiac events in patients with chronic renal disease not receiving dialysis. Kidney Int 2011;80:112-118.

[21] Hyun YY, Kim H, Oh KH, et al. eGFR and coronary artery calcification in chronic kidney disease. Eur J Clin Invest 2019:e13101.

[22] Hyun YY, Kim H, Oh KH, et al. Arterial stiffness as a risk factor for subclinical coronary artery calcification in predialysis chronic kidney disease: from the KNOW-CKD Study. Kidney Blood Press Res 2019;44:426-434.

[23] Ryu SR, Park SK, Jung JY, et al. The prevalence and management of anemia in chronic kidney disease patients: result from the KoreaN Cohort Study for Outcomes in Patients With Chronic Kidney Disease (KNOW-CKD). J Korean Med Sci 2017;32:249-256.

[24] Nemeth E, Tuttle MS, Powelson J, et al. Hepcidin regulates cellular iron efflux by binding to ferroportin and inducing its internalization. Science 2004;306:2090-2093. 
[25] Lee SW, Kim JM, Lim HJ, et al. Serum hepcidin may be a novel uremic toxin, which might be related to erythropoietin resistance. Sci Rep 2017;7:4260.

[26] Lee SW, Kim YH, Chung W, et al. Serum hepcidin and iron indices affect anemia status differently according to the kidney function of non-dialysis chronic kidney disease patients: Korean Cohort Study for Outcome in Patients With Chronic Kidney Disease (KNOW-CKD). Kidney Blood Press Res 2017;42:1183-1192.

[27] Kim CS, Bae EH, Ma SK, et al. Chronic kidney diseasemineral bone disorder in Korean patients: a report from the KoreaN Cohort Study for Outcomes in Patients With Chronic Kidney Disease (KNOW-CKD). J Korean Med Sci 2017;32:240-248.

[28] Kim HJ, Kang E, Oh YK, et al. The association between soluble klotho and cardiovascular parameters in chronic kidney disease: results from the KNOW-CKD study. BMC Nephrol 2018;19:51.

[29] Kim CS, Bae EH, Ma SK, et al. Association of serum osteoprotegerin levels with bone loss in chronic kidney disease: insights from the KNOW-CKD Study. PLoS One 2016;11: e0166792.

[30] Min HK, Sung SA, Oh YK, et al. Hepcidin, iron indices and bone mineral metabolism in non-dialysis chronic kidney disease. Nephrol Dial Transplant 2020;35:147-154.

[31] Nam KH, Kim H, An SY, et al. Circulating fibroblast growth factor-23 levels are associated with an increased risk of anemia development in patients with nondialysis chronic kidney disease. Sci Rep 2018;8:7294.

[32] Mehta R, Cai X, Hodakowski A, et al. Fibroblast growth factor 23 and anemia in the chronic renal insufficiency cohort study. Clin J Am Soc Nephrol 2017;12:1795-1803.

[33] Carrero JJ, Stenvinkel P, Cuppari L, et al. Etiology of the protein-energy wasting syndrome in chronic kidney disease: a consensus statement from the International Society of Renal Nutrition and Metabolism (ISRNM). J Ren Nutr 2013;23:77-90.

[34] Hyun YY, Lee KB, Han SH, et al. Nutritional status in adults with predialysis chronic kidney disease: KNOW-CKD Study. J Korean Med Sci 2017;32:257-263.

[35] Fouque D, Kalantar-Zadeh K, Kopple J, et al. A proposed nomenclature and diagnostic criteria for protein-energy wasting in acute and chronic kidney disease. Kidney Intc 2008;73:391-398.

[36] Iyasere O, Brown EA. Determinants of quality of life in advanced kidney disease: time to screen? Postgrad Med J
2014;90:340-347.

[37] Oh TR, Kim CS, Bae EH, et al. Association between vitamin $\mathrm{D}$ deficiency and health-related quality of life in patients with chronic kidney disease from the KNOW-CKD study. PLoS One 2017;12:e174282.

[38] Sung SA, Hyun YY, Lee KB, et al. Sleep duration and healthrelated quality of life in predialysis CKD. Clin J Am Soc Nephrol 2018;13:858-865.

[39] Hyun YY, Lee KB, Chung W, et al. Body mass index, waist circumference, and health-related quality of life in adults with chronic kidney disease. Qual Life Res 2019;28:10751083.

[40] Yun HR, Kim H, Park JT, et al. Obesity, metabolic abnormality, and progression of CKD. Am J Kidney Dis 2018;72: 400-410.

[41] Kang M, Kang E, Ryu H, et al. Measured sodium excretion is associated with CKD progression: results from the KNOWCKD study. Nephrol Dial Transpl 2020 in press.

[42] Lee SW, Han SH, Yoo TH, et al. Relationship between brachial-ankle and heart-femoral pulse wave velocities and the rapid decline of kidney function. Sci Rep 2018;8:821.

[43] Maroni BJ, Steinman TI, Mitch WE. A method for estimating nitrogen intake of patients with chronic renal failure. Kidney Int 1985;27:58-65.

[44] Lee SW, Kim YS, Kim YH, et al. Dietary protein intake, protein energy wasting, and the progression of chronic kidney disease: analysis from the KNOW-CKD study. Nutrients 2019;11:121.

[45] Oh TR, Choi HS, Kim CS, et al. Hyperuricemia has increased the risk of progression of chronic kidney disease: propensity score matching analysis from the KNOW-CKD study. Sci Rep 2019;9:6681.

[46] Lee MJ, Chang TI, Lee J, et al. Urine osmolality and renal outcome in patients with chronic kidney disease: results from the KNOW-CKD. Kidney Blood Press Res 2019;44: 1089-1100.

[47] Joo YS, Kim J, Park CH, et al. Urinary chloride concentration and progression of chronic kidney disease: results from the KoreaN cohort study for Outcomes in patients With Chronic Kidney Disease. Nephrol Dial Transplant 2019:gfz247.

[48] Kim HW, Park JT, Yoo TH, et al. Urinary potassium excretion and progression of CKD. Clin J Am Soc Nephrol 2019; 14:330-340.

[49] Keith DS, Nichols GA, Gullion CM, Brown JB, Smith DH. Longitudinal follow-up and outcomes among a population 
with chronic kidney disease in a large managed care organization. Arch Intern Med 2004;164:659-663.

[50] Tanaka K, Watanabe T, Takeuchi A, et al. Cardiovascular events and death in Japanese patients with chronic kidney disease. Kidney Int 2017;91:227-234.

[51] Orlandi PF, Huang J, Fukagawa M, et al. A collaborative, individual-level analysis compared longitudinal outcomes across the International Network of Chronic Kidney Disease (iNETCKD) cohorts. Kidney Int 2019;96:1217-1233.

[52] Han JS, Lee MJ, Park KS, et al. Albuminuria as a risk factor for anemia in chronic kidney disease: result from the KoreaN Cohort Study for Outcomes in Patients With Chronic Kidney Disease (KNOW-CKD). PLoS One 2015;10:e0139747.

[53] Yoon CY, Kim YL, Han SH, et al. Hypoadiponectinemia and the presence of metabolic syndrome in patients with chronic kidney disease: results from the KNOW-CKD study. Diabetol Metab Syndr 2016;8:75.

[54] Hyun YY, Lee KB, Oh KH, et al. Serum adiponectin and protein-energy wasting in predialysis chronic kidney disease. Nutrition 2017;33:254-260.

[55] Kim HY, Bae EH, Ma SK, et al. Association of serum adiponectin level with albuminuria in chronic kidney disease patients. Clin Exp Nephrol 2016;20:443-449.

[56] Kim CS, Bae EH, Ma SK, et al. Association of serum adiponectin concentration with aortic arterial stiffness in chronic kidney disease: from the KNOW-CKD study. Clin Exp Nephrol 2017;21:608-616.

[57] Kim H, Yun HR, Park S, et al. High serum adiponectin is associated with anemia development in chronic kidney disease: the results from the KNOW-CKD study. Cytokine 2018;103:1-9.

[58] Song SH, Oh TR, Choi HS, et al. High serum adiponectin as a biomarker of renal dysfunction: results from the KNOWCKD study. Sci Rep 2020;10:5598.

[59] Hyun YY, Kim H, Oh YK, et al. High fibroblast growth factor 23 is associated with coronary calcification in patients with high adiponectin: analysis from the KoreaN cohort study for Outcome in patients With Chronic Kidney Disease (KNOW-CKD) study. Nephrol Dial Transplant 2019;34:123129.

[60] Nam KH, Chang TI, Joo YS, et al. Association between serum high-density lipoprotein cholesterol levels and pro- gression of chronic kidney disease: results from the KNOWCKD. J Am Heart Assoc 2019;8:e011162.

[61] Kang E, Ryu H, Kim J, et al. Association between high-sensitivity cardiac troponin $\mathrm{T}$ and echocardiographic parameters in chronic kidney disease: results from the KNOWCKD Cohort Study. J Am Heart Assoc 2019;8:e013357.

[62] Kim HJ, Lee J, Chae DW, et al. Serum klotho is inversely associated with metabolic syndrome in chronic kidney disease: results from the KNOW-CKD study. BMC Nephrol 2019;20:119.

[63] Chae SY, Chung W, Kim YH, et al. The correlation of serum osteoprotegerin with non-traditional cardiovascular risk factors and arterial stiffness in patients with pre-dialysis chronic kidney disease: results from the KNOW-CKD Study. J Korean Med Sci 2018;33:e322.

[64] Hyun YY, Kim H, Sung SA, et al. Association between urine creatinine excretion and arterial stiffness in chronic kidney disease: data from the KNOW-CKD Study. Kidney Blood Press Res 2016;41:527-534.

[65] Koo H, Hwang S, Kim TH, et al. The ratio of urinary sodium and potassium and chronic kidney disease progression: results from the KoreaN Cohort Study for Outcomes in Patients with Chronic Kidney Disease (KNOW-CKD). Medicine (Baltimore) 2018;97:e12820.

[66] Kim H, Park S, Jhee JH, et al. Urinary angiotensinogen level is associated with potassium homeostasis and clinical outcome in patients with polycystic kidney disease: a prospective cohort study. BMC Nephrol 2019;20:104.

[67] Muntner P, Newsome B, Kramer H, et al. Racial differences in the incidence of chronic kidney disease. Clin J Am Soc Nephrol 2012;7:101-107.

[68] Mehrotra R, Kermah D, Fried L, Adler S, Norris K. Racial differences in mortality among those with CKD. J Am Soc Nephrol 2008;19:1403-1410.

[69] Dienemann T, Fujii N, Orlandi P, et al. International Network of Chronic Kidney Disease cohort studies (iNET-CKD): a global network of chronic kidney disease cohorts. $B M C$ Nephrol 2016;17:121.

[70] Alencar de Pinho N, Levin A, Fukagawa M, et al. Considerable international variation exists in blood pressure control and antihypertensive prescription patterns in chronic kidney disease. Kidney Int 2019;96:983-994. 